\title{
Custo/benefício de aeronaves: uma abordagem pela Análise Envoltória de Dados
}

\author{
Rafael lglesias Reinas ${ }^{\mathrm{a} *}$, Enzo Barberio Mariano ${ }^{\mathrm{b}}$, \\ Daisy Aparecida do Nascimento Rebelatto ${ }^{c}$ \\ a*raf.rei@ibest.com.br, EESC-USP, Brasil \\ benzo.mariano@gmail.com, EESC-USP, Brasil \\ 'daisy@sc.usp.br, EESC-USP, Brasil
}

\begin{abstract}
Resumo
0 objetivo deste artigo é a aplicação da técnica de Pesquisa Operacional Análise Envoltória de Dados - DEA, por meio do índice composto das fronteiras clássica e invertida, para avaliar o custo/beneficio de aeronaves de transporte civil. Para tal, foram adotados como inputs: a) preço de mercado, e b) custos operacionais; e como outputs: a) peso de carga paga, b) velocidade de cruzeiro, e c) razão máxima de subida com um único motor. Para garantir a homogeneidade das DMUs, os aviões foram divididos segundo o sistema de propulsão e segundo as categorias: regional, narrow-body e wide-body. Foram também agrupados em diferentes alcances, para que se pudessem identificar os aviões com melhor custo/beneficio em cada um. Os resultados encontrados permitem afirmar que os aviões com o melhor custo/beneficio são os que já tiveram sua produção descontinuada, mas que ainda não estão muito defasados tecnologicamente.
\end{abstract}

Palavras-chave

Aeronaves de transporte civil. Análise custo/beneficio. Análise Envoltória de Dados - DEA. Fronteira invertida.

\section{Introdução}

Uma companhia aérea pode expandir sua frota de duas formas: por meio de aquisição direta ou pela prática de leasing, que é um contrato de locação. Tanto as empresas de leasing aeronáutico quanto às companhias aéreas com frota própria, precisam constantemente conhecer o custo/benefício dos aviões que vão adquirir, seja para alugá-los, seja para utilizá-los em suas operações.

Do mesmo modo, é igualmente importante que as empresas fabricantes de aeronaves conheçam o custo/ benefício de seus modelos, visto que isso possibilita que elas se posicionem melhor no mercado, além de terem mais parâmetros para a tomada de decisão. Pode-se dizer, portanto, que é bastante conveniente que existam métodos simples, rápidos e eficazes para avaliar o custo/benefício de aeronaves.

A tarefa de uma aeronave de transporte civil é levar uma carga, ou um grupo de passageiros, de um local para outro por meio de uma rota. Um comprador, de modo geral, adquire seus aviões para um tipo específico de rota, sendo que muitas vezes o avião mais adequado para uma pode não ser para outra. Vale ressaltar que o que caracteriza e diferencia uma rota é o seu alcance, que pode ser definido como a distância entre o local de decolagem e o local de pouso.

0 objetivo desta pesquisa foi a determinação da eficiência, calculada a partir da relação custo/ benefício, de um conjunto de aeronaves de transporte civil de passageiros e cargas, para diversos níveis de alcance. Espera-se que esse artigo possa auxiliar, em primeiro lugar, o processo de tomada de decisão de fabricantes de aeronaves, ajudando-os a decidir, por exemplo, sobre a descontinuação ou investimento em determinado modelo ou sobre a necessidade de inovação; espera-se também que o artigo possa servir como um guia para que possíveis compradores possam verificar quais são as aeronaves que lhes trarão, dadas as suas necessidades, o melhor custo/ benefício. 
A abrangência desse objetivo requereu que fosse utilizada uma técnica que permitisse um conjunto de análises maior do que uma simples escolha entre alternativas; para tal, foi selecionada a técnica de Pesquisa Operacional Análise Envoltória de Dados (Data Envelopment Analysis - DEA). A DEA foi, originalmente, elaborada para analisar relações de produção entre insumos e produtos, porém, com o tempo, acabou sendo adaptada para utilizações não convencionais.

Entre as principais abordagens não convencionais da DEA está a análise custo/benefício, que foi utilizada por Kusomanen, Bijsterbosch e Dellink (2009) para avaliar o problema de emissões de gás carbônico. Segundo Womer et al. (2006), que desenvolveu um modelo de custo-benefício por meio da DEA, nesse tipo de aplicação os custos representarão os insumos e os benefícios, os produtos.

Além da DEA, outras técnicas poderiam ser utilizadas para avaliar o custo/benefício de aeronaves, tais como a lógica fuzzy (WANG; CHANG, 2007) e as técnicas multicritério de apoio à decisão, das quais se pode destacar: a problemática alfa do método ELECTRE (MAYSTRE; PICTET; SIMOS, 1994), a AHP e a PROMÉTHÉE (RANGEL; GOMES, 2010). A DEA terá como vantagens em relação a essas técnicas: a) o fornecimento de um índice de eficiência que agrega variáveis com unidades de medida totalmente diferentes; b) a não necessidade de informações prévias sobre o peso de cada variável; c) o poder de comparar, de forma rápida, uma enorme quantidade de alternativas ao mesmo tempo; e d) a possibilidade de realização de uma série de outras análises, que não fazem parte do escopo desse artigo, tais como, por exemplo, a determinação de metas para que os aviões se tornem eficientes.

\section{DEA no setor aeronáutico}

Foram encontrados na literatura vários trabalhos que aplicam a DEA ao setor aeronáutico, como, por exemplo, alguns que contemplam a avaliação de aeroportos, tais como: a) Parker (1999), que avaliou aeroportos da Inglaterra; b) Abbott e Wu (2002), que avaliaram aeroportos da Austrália; c) Martin e Roman (2006), que avaliaram aeroportos da Espanha; d) Bazargan e Vasigh (2003), que avaliaram aeroportos americanos; e) Yoshida e Fujimoto (2004), que avaliaram aeroportos japoneses; f) $\mathrm{Yu}$ (2010), que avaliou aeroportos de Taiwan; g) Roghanian e Foroughi (2010), que avaliaram aeroportos iranianos; h) Lam, Low e Tang (2009), que avaliaram aeroportos asiáticos; i) Lin e Hong (2006), que avaliaram os 20 maiores aeroportos do mundo; ej) Guedes, Santos e Correia (2006), Pacheco e Fernandes (2003) e Almeida, Mariano e Rebelatto (2008), que avaliaram aeroportos brasileiros.

Quanto à avaliação da eficiência de companhias aéreas utilizando a DEA, podem-se citar os trabalhos de: a) Good et al. (1995), Higgins, Lawphongpanich e Mahoney (2008) e Ray (2008), que avaliaram companhias aéreas americanas; b) Barros e Peypoch (2009), que avaliaram companhias aéreas europeias; c) Distexhe e Parelman (1994), que avaliaram companhias aéreas da Ásia, Europa, América do Norte e Oceania; e d) Correia e Soares de Mello (2009), Silveira et al. (2008) e Soares de Mello et al. (2003), que avaliaram companhias aéreas brasileiras.

Também foi identificado o artigo de Bonacorsi, Giuri e Pierotti (2005), que propõe a aplicação da DEA para avaliar a eficiência de motores de avião.

\section{Análise Envoltória de Dados}

Uma Unidade Tomadora de Decisão (Decision Making Unit - DMU) pode ser definida como um sistema autônomo, que transforma um conjunto de entradas (inputs) em um conjunto de saídas (outputs), podendo designar empresas, unidades administrativas, pessoas ou até mesmo aviões.

AAnálise Envoltória de Dados (Data Envelopment Analysis - DEA) é uma técnica de Pesquisa Operacional que objetiva, por meio da construção empírica de uma fronteira, a determinação da eficiência de um conjunto de DMUs. A DEA pode ser descrita como um procedimento matemático, baseado em programação linear, capaz de encontrar o conjunto de pesos que maximiza a eficiência de uma DMU, o que permite a obtenção de um índice que incorpora múltiplos inputs e múltiplos outputs, sem a necessidade de convertê-los para uma base comum. Vale ressaltar que os resultados da DEA devem ser avaliados tendo como base o tipo de retorno à escala adotado e a orientação escolhida.

0 tipo de retorno à escala determina os dois principais modelos da DEA: a) o modelo CRS (Constant Returns to Scale - Retornos Constantes à Escala), também chamado de CCR (CHARNES; COOPER; RHODES, 1978); b) e o modelo VRS (Variable Returns to Scale - Retornos Variáveis à Escala), também chamado de BCC (BANKER; CHARNES; COOPER, 1984). 0 modelo CCR se baseia em uma relação linear, sendo que um aumento nos inputs provoca um aumento proporcional e constante nos outputs; já o modelo BCC considera que um aumento nos inputs ocasiona um aumento não necessariamente proporcional nos outputs.

A orientação, por sua vez, determina qual tipo de variável, input ou output, será utilizada como 
base para o cálculo da eficiência, sendo que: a) os modelos orientados aos inputs procuram responder, dado o atual nível de outputs, o quanto os inputs poderiam ser reduzidos; b) e os modelos orientados aos outputs procuram responder, dado o atual nível de inputs, o quanto os outputs poderiam ser aumentados (COOPER; SEIFORD; TONE, 2000).

$\mathrm{Na}$ análise presente neste artigo, foi utilizado o modelo BCC orientado aos inputs. A orientação aos inputs foi escolhida porque se procurou a máxima redução de custo, mantendo-se o atual nível de benefício. Já o modelo BCC foi escolhido porque não existe proporcionalidade entre os inputs e os outputs do modelo de custo/benefício adotado neste artigo.

\section{Fronteira invertida}

A fronteira eficiente, de acordo com as formulações originais da DEA, é denominada fronteira clássica, mas existe outra formulação, bastante interessante, que é denominada fronteira invertida. A fronteira invertida, que consiste simplesmente em trocar os inputs e outputs de lugar, foi uma das várias soluções que foram elaboradas para resolver o problema dos inúmeros empates de DMUs eficientes, que eram obtidos na DEA clássica (LETA et al., 2005). Vale ressaltar que esses empates são consequência do sistema de atribuição de pesos da DEA, que não leva em consideração, por exemplo, o equilíbrio entre as variáveis, as informações prévias sobre os pesos, a atribuição de pesos nulos, além de diversos outros fatores.

0 uso apenas da fronteira invertida, porém, não é suficiente para a discriminação de DMUs, sendo que para tal é necessário utilizar o índice composto. Segundo Leta et al. (2005), o índice composto é calculado a partir da média aritmética entre o índice da fronteira clássica $\left(\mathrm{E}_{\text {clássica }}\right)$ e o índice da fronteira invertida subtraído de um ( $1-E_{\text {invertida }}$ ), sendo que é interessante fazer a posterior normalização dos resultados para que se obtenha um índice que varie entre 0 e 1.

Como já mencionado, o índice composto é apenas um dos vários modelos existentes para a discriminação de DMUs dentro da metodologia DEA, sendo que eles podem ser divididos em: a) modelos que exigem informações prévias, tais como: DEA com restrições de peso, análise de valor e estrutura de preferências (ANGULO-MEZA; LINS, 2002); b) e modelos que não exigem informações prévias, tais como: modelo de supereficiência, índice composto e avaliação cruzada (SOARES DE
MELLO et al. 2008). Segundo Ângulo-Meza e Lins (2002), as principais desvantagens dos modelos que exigem informações prévias são a falta de consenso e a subjetividade. Deve-se dizer, porém, que esses modelos seriam indispensáveis caso a opinião dos compradores, que são os maiores interessados na determinação do custo/benefício de aeronaves, fosse levada em conta (ficará como recomendação para trabalhos futuros).

Pelo índice composto, a DMU mais eficiente é aquela que consegue ter um bom desempenho naquilo em que é melhor, dado pelo nível de eficiência clássica, sem ter um desempenho ruim naquilo em que é pior, dado pelo nível de eficiência invertida (LETA et al., 2005). Com isso, pode-se dizer que, dentre todos os modelos de discriminação citados, o índice composto é o mais adequado para a análise deste artigo, pois considera que todos os fatores em análise são importantes, e que nenhum pode ser relegado a segundo plano. Além disso, esse modelo apresenta procedimentos de cálculo relativamente simples e já se encontra implementado no software SIAD (ANGULO-MEZA et al., 2005).

\section{Categorias de aeronaves}

Neste artigo, os aviões comerciais foram classificados em categorias, o que possibilitou a comparação apenas de aeronaves que são concorrentes dentro de um determinado segmento (garantiu DMUs homogêneas). Existem diversas categorizações possíveis para aviões civis de transporte, que podem ser separados, por exemplo, pelo número de motores, forma de propulsão, autonomia, manobrabilidade, peso, dentre outros. No presente trabalho, as aeronaves foram classificadas de acordo com o sistema de propulsão (turboélice ou jato) e o tamanho, sendo definidas as categorias:

1. Aviões Regionais: São aeronaves de pequena dimensão, tendo entre 30 e 110 lugares. É a única categoria que compartilha aviões turboélice e a jato.

2. Aviões Narrow-body: São aeronaves de médio porte, com diâmetro de fuselagem variando de 3 a 4 metros, assentos ordenados de 2 a 6 fileiras e um único corredor. A capacidade típica destes aviões varia de 110 a 250 passageiros.

3. Aviões Wide-body: São aeronaves de grande porte, com diâmetro de fuselagem variando de 5 a 6 metros, poltronas organizadas de 7 a 10 fileiras e 2 corredores. A capacidade típica desses aviões varia de 250 a 600 passageiros. 


\section{Pesos de um avião}

Antes de comentar mais especificamente sobre as variáveis de input e output, é interessante realizar uma pequena introdução sobre os diferentes pesos que compõem uma aeronave e que constituem a base para a determinação de algumas das variáveis utilizadas neste artigo:

a) Peso de carga paga (payload): é o peso dos passageiros e/ou cargas, que são levados de um local para outro.

b) Peso de combustível: é a quantidade de combustível que é carregada no avião para uma determinada viagem.

c) Peso da aeronave vazia (Operating Empty Weight $O E W$ ): é a soma de todos os pesos que possibilitam o voo do avião; é o peso da aeronave sem contar a carga paga e o combustível, mas incluindo os equipamentos e a tripulação.

d) Peso de decolagem (Take-off Weight - TOW): é o peso do avião no momento da decolagem; é dado pela soma do peso da aeronave vazia, com o peso de combustível e o payload.

e) Peso de pouso (Landing Weight - $L W$ ): é o peso da aeronave no momento do pouso; é dado pelo peso de decolagem menos o peso de combustível gasto.

f) Peso máximo de carga paga (Maximum Structural Payload - MSP): é o limite de payload que um avião pode transportar; é limitado por questões estruturais.

g) Peso máximo de combustível (Maximum Fuel Load - MFL): é a máxima quantidade de combustível que cabe nos tanques de um avião.

h) Peso máximo de decolagem (Maximum Take-off Weight - MTOW): é o maior peso com que um avião é capaz de decolar; é o peso limite em que a estrutura da aeronave consegue suportar todos os esforços a que é submetida na decolagem.

i) Peso máximo de pouso (Maximum Landing Weight - $M L W$ ): é o maior peso com que uma aeronave consegue pousar; é determinado pela máxima energia que pode ser absorvida pelo trem de pouso.

\section{Modelo de custo/benefício}

Pela perspectiva de análise adotada neste artigo, as variáveis de input mais relevantes, segundo consulta a especialistas da área de engenharia aeronáutica, são: a) custos operacionais diretos e b) preço de mercado; já as variáveis de output mais relevantes, também segundo consulta a especialistas, são: a) payload máximo, b) velocidade de cruzeiro
Quadro 1. Sites consultados.

\begin{tabular}{|c|c|}
\hline Site & Endereço eletrônico \\
\hline Boeing (2009) & www.boeing.com \\
\hline Embraer (2009) & www.embraer.com \\
\hline Airbus (2009) & www.airbus.com \\
\hline Bombardier (2009) & www.bombardier.com \\
\hline Avion Safety Network (2009) & www.aviation-safety.net \\
\hline Whel (2009) & www.whel.de \\
\hline Risingup Aviation Resources (2009) & www.risingup.com \\
\hline
\end{tabular}

e c) razão máxima de subida com um único motor. Vale ressaltar que, sob o ponto de vista adotado, todas essas variáveis são discricionárias, visto que dependem de decisões de projeto.

0 custo operacional direto e o payload são dependentes do alcance, sendo que foram extraídos de um conjunto de curvas, que os relacionam a essa variável; os outros fatores, por sua vez, são constantes para uma determinada aeronave e independem do alcance estabelecido. A variável "razão de subida com um motor inoperante" foi estimada a partir das equações da teoria da dinâmica de voo; os outros fatores, por outro lado, foram extraídos diretamente dos sites dos fabricantes ou de sites de aviação, tais como os mostrados no Quadro 1.

As variáveis selecionadas estão relacionadas aos atributos que são mais procurados em aeronaves, que são: economia, capacidade, velocidade, confiabilidade e segurança. Como todos esses quesitos são considerados imprescindíveis e como existe uma boa disponibilidade de DMUs, não houve a preocupação em se realizar uma seleção mais criteriosa das variáveis, utilizando, por exemplo, os métodos Stepwise ou multicritério, que estão expressos em Senra et al. (2007).

Ao se realizar uma análise de correlação simples, porém, verificou-se que nenhum dos outputs e nenhum dos inputs apresentou sinal de redundância (alta correlação); também se verificou que os dois inputs apresentaram boas correlações com pelo menos um dos outputs, o que é um indicativo de que pode existir uma relação de causalidade entre eles.

\subsection{Custos Operacionais Diretos (DOC)}

Os Custos Operacionais Diretos (DOC) abrangem os custos de propriedade, operação e manutenção de uma aeronave, sendo que dependem principalmente do número de assentos e do alcance. Segundo Roskan (1979), os resultados do DOC, apesar de serem frequentemente questionados quanto 
à magnitude, podem ser utilizados de maneira bastante satisfatória para fazer a comparação de aeronaves, pois é utilizada uma base de cálculo comum.

Segundo Fielding (2003), o custo de propriedade de uma aeronave é composto pelos custos de depreciação, financiamento e seguro, sendo que: a depreciação está relacionada com o número de voos realizados e com o tempo de utilização do avião; o custo do financiamento está relacionado com a taxa de juros estipulada e com o valor financiado; e o custo do seguro é baseado no preço inicial da aeronave.

Os custos de operação de uma aeronave, por sua vez, se referem aos gastos com combustível e tripulação. Para o cálculo do custo de combustível, basta multiplicar a quantidade utilizada pelo preço unitário. Já para o custo da tripulação, deve-se multiplicar o número de horas de voo por uma constante denominada "tripulação-\$/hora", que depende do número de funcionários em voo e da qualidade do atendimento aos clientes (FIELDING, 2003).

Para finalizar, há também o custo de manutenção, que é um dos maiores itens do DOC, e que comumente tem seus gastos bem definidos, sendo que a maior quantia é gasta na conservação da estrutura básica e do sistema motopropulsor da aeronave (FIELDING, 2003).

Diversas instituições criaram métodos para estimar o DOC, sendo que o utilizado nesta pesquisa foi desenvolvido pela Universidade Técnica de Berlim e estipula os custos de acordo com uma série de constantes relacionadas a quatro pesos inerentes à aeronave: a) peso de combustível, ligado ao custo de combustível; b) peso máximo de decolagem (MTOW), ligado ao custo de manutenção; c) payload, ligado ao custo de tripulação; d) e peso da aeronave vazia (OEW), ligado ao custo de propriedade. Vale ressaltar, porém, que o DOC já é fornecido pelo fabricante na sua forma agregada.

0 fato de o payload ser um dos fatores considerados para estimar o DOC causou problemas no modelo de custo/benefício deste artigo, já que o payload é também um dos outputs da análise. Sendo assim, o payload deveria entrar na análise ao mesmo tempo como output, que deve ser aumentado, e input, que deve ser reduzido, o que não faz sentido. Para contornar esse problema, deve-se estabelecer a condição de que o payload é constante e que, portanto, a redução do DOC pode ser realizada via qualquer fator, menos alterando o payload. Como a análise deste artigo é orientada para a minimização dos inputs, supor o payload constante é coerente, a não ser para as DMUs em que haja folga nessa variável.

Deve-se ressaltar que nos casos em que haja folga no payload, será introduzido um pequeno viés nos resultados, pois o DOC ficará subestimado, visto que o aumento sugerido para o payload aumentará o DOC e isso não estará sendo considerado na análise. Esse viés, porém, não comprometerá os resultados, já que as folgas são pequenas e raras e 0 custo da tripulação representa apenas uma pequena parcela do DOC.

\subsection{Payload}

0 payload é um dos outputs mais relevantes na análise deste artigo, já que se relaciona com o objetivo primário de uma aeronave, que é transportar o máximo possível de passageiros e/ ou encomendas. 0 payload está diretamente relacionado com o alcance, sendo que essa relação pode ser representada em um diagrama, que é disponibilizado pelo fabricante da aeronave.

0 diagrama payload-alcance expressa 0 carregamento máximo que uma aeronave pode transportar de forma segura, dado um alcance; ele é baseado na hipótese de que a aeronave vai sempre levar o máximo payload possível, com o mínimo peso de combustível para que a rota seja cumprida com segurança (PADILHA, 1996). A Figura 1 exemplifica um desses diagramas.

A linha AD do diagrama da Figura 1 representa o peso de decolagem (TOW) de uma aeronave. Esse peso é composto pelo peso da aeronave vazia (OEW) somado com o payload e o peso de combustível. Vale ressaltar que o alcance é diretamente dependente da quantidade de combustivel e inversamente dependente do TOW do avião.

A partir da situação de alcance zero até o ponto B do diagrama, é possível aumentar o alcance, via aumento de combustível, sem sacrificar o payload, que pode ser utilizado em seu valor limite (MSP). Ao chegar ao ponto B, porém, o peso de decolagem da aeronave atinge o valor máximo (MTOW), sendo que, para obter um alcance maior, parte do payload deverá ser substituída por peso de combustível. Vale ressaltar que quanto maior a quantidade de combustível, menor será o payload que poderá ser levado. Essa situação persiste até o ponto C, quando o peso de combustível atinge sua capacidade máxima (MFL) e a única forma de se aumentar o alcance é pela diminuição do TOW, sendo que para isso deve-se reduzir, a uma taxa ainda mais elevada, o payload do avião. 


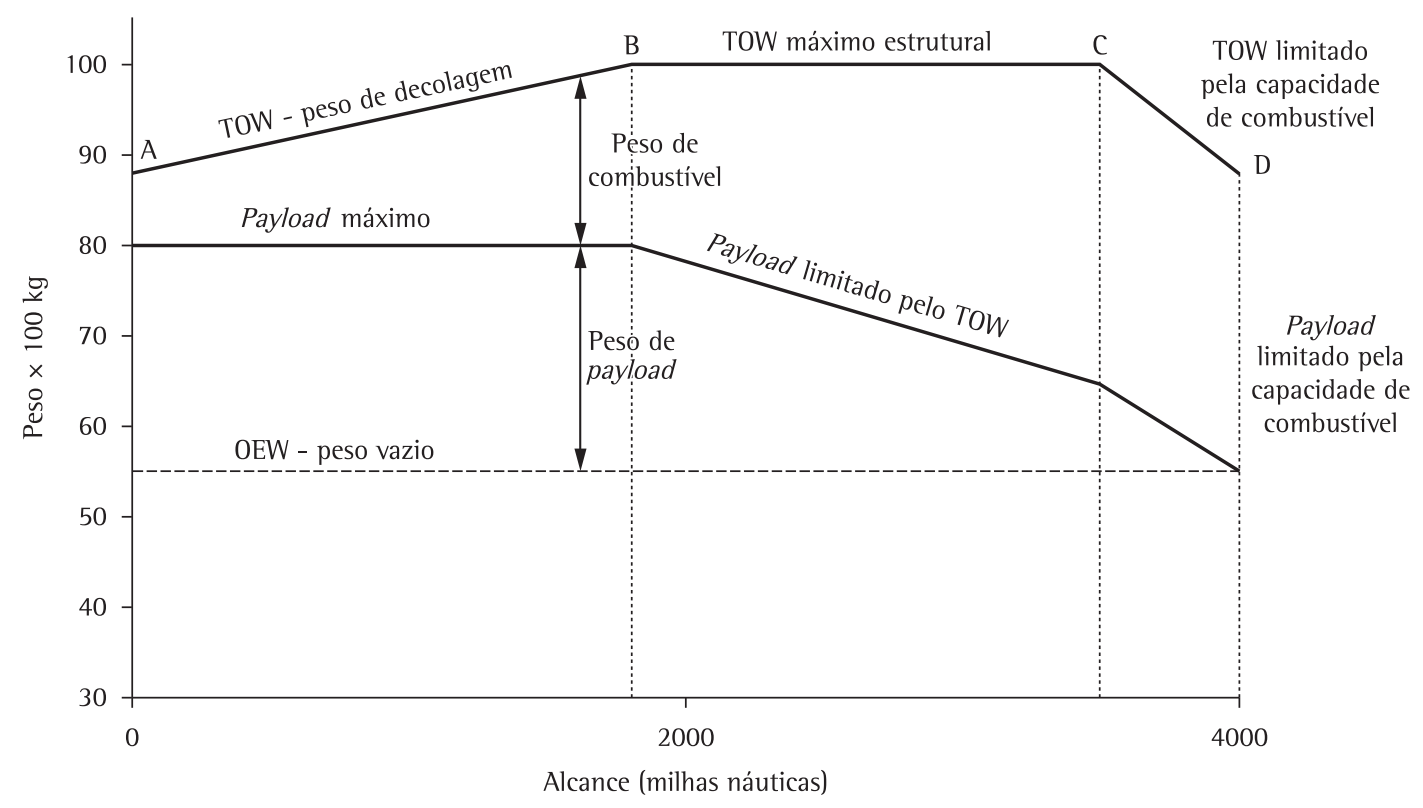

Figura 1. Diagrama payload-alcance.

Nenhuma empresa aérea, especialmente as que trabalham com aeronaves pequenas, tem interesse em operar com grandes alcances (que fiquem entre os pontos C e D do diagrama), pois o payload seria muito pequeno. Do mesmo modo, aviões de grande porte não são desejáveis para cumprir missões de curto alcance, pois podem chegar ao destino pesando mais do que o seu peso máximo de pouso (MLW). Essas considerações foram levadas em conta na pesquisa, de modo que aviões de grande porte não foram alocados para rotas de curto alcance e aviões de pequeno porte não foram alocados para rotas com alcance muito alto.

\subsection{Velocidade de cruzeiro}

A velocidade é um item muito importante em uma análise de custo/benefício de aeronaves, pois a rapidez com que um avião consegue cumprir sua rota pode trazer muitas vantagens para as companhias aéreas, tanto no que se refere a custos mais baixos, quanto no que se refere ao aumento da satisfação dos clientes, que chegam mais rápido aos seus destinos.

0 cruzeiro é a etapa de voo compreendida entre o fim da subida e o início da descida e é onde o avião passa a maior parte do tempo de uma viagem; sendo assim, a velocidade de cruzeiro é a mais importante para ser analisada, já que as outras velocidades (de decolagem, subida, descida e pouso) têm baixo impacto no tempo total de voo. A velocidade de cruzeiro reflete a capacidade do motor da aeronave de transformar a energia provida da combustão em energia cinética, sendo que essa capacidade é dependente da potência disponível no motor, da altitude do voo, do peso e da aerodinâmica da aeronave (PADILHA, 1996).

Por meio de equações da dinâmica de voo, pode-se calcular, partindo da hipótese de que o avião está utilizando o payload máximo e que está voando a altitudes que otimizam o seu desempenho, a velocidade de cruzeiro que maximiza o alcance de um avião. Essa velocidade é denominada de "Velocidade de Máximo Alcance" (Maxi Range Speed - MRS) e garante a maior economia de combustivel.

Apesar de a MRS minimizar o consumo de combustível, o valor absoluto desta velocidade é muito baixo para as pretensões das companhias aéreas. Foi verificado, porém, que, ao se aumentar o valor da MRS de 3 a 5\%, o consumo de combustível aumentava apenas $1 \%$, sendo que esse preço pago pela velocidade adicional é considerado pequeno em comparação às vantagens trazidas. Esta velocidade ajustada é denominada de "Velocidade de Longo Alcance" (Long Range Speed - LRS) e é a mais utilizada pelas companhias aéreas, sendo que foi adotada como output desta análise. 


\subsection{Razão de subida com um motor inoperante}

Mostrou-se conveniente adicionar à análise uma variável ligada à segurança no voo que refletisse a confiabilidade da aeronave diante de uma situação emergencial. Para tal, foi incluída no modelo a razão de subida (ou velocidade vertical) com um motor inoperante.

Na situação hipotética que contextualiza essa variável, o avião, com peso máximo de decolagem, deve tentar subir à altitude de cruzeiro com um de seus motores não funcionando. Nessa situação, quanto mais rápido uma aeronave conseguir alcançar a altitude de cruzeiro, ou seja, quanto

Quadro 2. Quantidade de aviões por alcance.

\begin{tabular}{|c|c|c|c|}
\hline Propulsão & Categoria & $\begin{array}{c}\text { Alcance } \\
(\mathrm{km})\end{array}$ & $\begin{array}{c}\text { Quantidade de } \\
\text { aeronaves }\end{array}$ \\
\hline \multirow{3}{*}{ Turboélice } & \multirow{3}{*}{ Regional } & 500 & 21 \\
\hline & & 1000 & 21 \\
\hline & & 1500 & 17 \\
\hline \multirow{11}{*}{ Jato } & \multirow{2}{*}{ Regional } & 1000 & 24 \\
\hline & & 2500 & 18 \\
\hline & \multirow{3}{*}{ Narrow-body } & 2500 & 32 \\
\hline & & 4000 & 33 \\
\hline & & 5500 & 16 \\
\hline & \multirow{6}{*}{ Wide- body } & 4000 & 24 \\
\hline & & 5500 & 35 \\
\hline & & 7000 & 41 \\
\hline & & 8500 & 36 \\
\hline & & 10000 & 28 \\
\hline & & 11500 & 20 \\
\hline
\end{tabular}

maior for a sua razão de subida, mais segura ela será. A razão de subida com um motor inoperante representa a reserva de potência do motor da aeronave.

\section{Método}

Como já mencionado, neste artigo foi utilizada a técnica DEA, por meio da ferramenta computacional SIAD (ANGULO-MEZA et al., 2005), com o objetivo de avaliar o custo/beneficio, para diversos alcances, de um conjunto de aeronaves de diferentes categorias, modelos e fabricantes. Foram analisados 127 aviões, sendo 21 turboélices, 32 jatos regionais, 33 jatos narrow-body e 41 jatos wide-body.

Para cada um dos alcances, estabelecido em cada categoria, foi realizada uma DEA diferente, totalizando 14 aplicações. Vale ressaltar que cada aeronave de uma determinada categoria poderia ser analisada considerando-se diversos alcances, contanto que eles fossem viáveis. 0 Quadro 2 mostra quais foram os alcances estabelecidos e quantos aviões de cada categoria entraram na análise em cada um.

Pode-se notar, pelo Quadro 2, que em todas as aplicações da DEA houve uma quantidade de DMUs superior ao triplo da soma do número de inputs com o número de outputs (superior a 15); isso significa que, segundo critérios estabelecidos empiricamente por Charnes et al. (2000), todas as 14 análises DEA foram consistentes.

Quadro 3. Quantidade de aviões por Fabricantes.

\begin{tabular}{|c|c|c|c|}
\hline Fabricante & Prefixos & Nacionalidade & Quantidade de aeronaves \\
\hline Aerei da Trasporto Regionale & ATR & ltália e França & 3 \\
\hline Airbus & A & Pan-europeia & 20 \\
\hline Antonov & AN & Ucrânia & 33 \\
\hline Boeing & B & Cstados Unidos & 10 \\
\hline Bombardier & CRJ e Dash & Inglaterra & 2 \\
\hline British Aerospace & Avro, BAe e Jetstream & Inglaterra & 9 \\
\hline British Aircraft Corporation & BAC & Brasil & 3 \\
\hline Embraer & E, EMB e ERJ & Estados Unidos & 3 \\
\hline Fairchild Dornier & FD & Holanda & 2 \\
\hline Fokker & F & Estados Unidos & 6 \\
\hline Beechcraft & Beech & Eśssia & 14 \\
\hline llyushin & llados Unidos & 2 \\
\hline Lockheed & L & Estados Unidos & 2 \\
\hline McDonnell Douglas & MD e DC & Suécia & 1 \\
\hline Saab & Saab & Rússia & \\
\hline Tupolev & TU & República Checa & \\
\hline Turbolet & LET & Cússia & \\
\hline Yakovlev & Yak & China & 2 \\
\hline Xi'an & Y & \\
\hline
\end{tabular}


Foram avaliadas aeronaves de 14 nacionalidades e de 19 fabricantes, que podem ser encontrados no Quadro 3.

\section{Análise de resultados}

Nesse tópico serão apresentados os resultados, separados por categorias, da eficiência composta normalizada de cada avião, para vários níveis de alcance.

A Tabela 1 mostra o resultado da eficiência composta normalizada para aviões turboélices. Como pode ser observado nessa tabela, foi verificado, entre os turboélices, um ótimo desempenho dos aviões Dash 8-100A, da empresa canadense Bombardier, e Beech 1900D, da empresa americana Beechcraft, que foi o avião mais eficiente em todos os alcances. 0 Beech 1900D é um avião de pequeno porte, com capacidade para até 20 passageiros e que teve a sua fabricação descontinuada em 2002. Os aviões Y-7-200A, Jetstream 41 e Saab 340B+, foram os aviões turboélices com pior custo/benefício em todos os alcances.

A Tabela 2 mostra o resultado da eficiência composta normalizada para jatos regionais. Pelo que se pode notar dessa tabela, o jato regional com melhor custo/benefício, considerando os dois alcances, é o F 70, que é uma aeronave com capacidade para 79 passageiros, que teve sua produção interrompida em 1997. De modo geral, tanto o F70 quanto o F 100, que são da fabricante holandesa Fokker, tiveram excelentes resultados, muito superiores aos das demais aeronaves. 0 jato regional com o pior desempenho foi o Yak 42, da empresa russa Yakovlev.

A Tabela 3 mostra a eficiência composta normalizada para jatos do tipo Narrow-body. Como se pode verificar nessa tabela, os aviões narrow-body que apresentaram maior nível de eficiência foram o TU 154M, para o alcance de $2500 \mathrm{~km}$, o B 757-200F, para o alcance de $4000 \mathrm{~km}$ e o B 757-200, para o alcance de $5500 \mathrm{~km}$. Vale ressaltar que o B 757-200 não apresentou bom desempenho para baixos alcances, se destacando apenas no de $5500 \mathrm{~km}$. Tanto o B 757-200F, que é um avião de carga, quanto o B 757-200, que é a sua versão para passageiros, tiveram sua produção interrompida em 2004, sendo que eram fabricados pela Boeing; já o TU 154M, que é um avião da empresa russa Tupolev, parou de ser fabricado em 2006. Os aviões narrow-body com os piores desempenhos, em todos os alcances, foram o A 300-600, o A 300-600ST (Beluga) e o B 737-200, sendo que os dois primeiros são da Airbus e o último da Boeing.

A Tabela 4 mostra a eficiência composta normalizada para jatos do tipo wide-body. Pelo que se encontra expresso nessa tabela, o avião widebody de melhor custo/beneficio foi o MD 11F, que foi o mais eficiente em todos os alcances. O MD11F é uma aeronave de carga, da fabricante americana McDonnell Douglas, que teve sua produção descontinuada em 2000. Os aviões com as piores eficiências foram o B 777-300 e o A 380-800.

0 Quadro 4 mostra os aviões mais e menos eficientes de cada categoria, para cada alcance estabelecido. 0 que pode ser ressaltado a partir desse quadro é que: a) dentre os aviões turboélices, o que mais se destacou foi o Beech 1900D da Beechcraft, sendo que o avião Dash 8-100A da Bombardier também merece algum destaque; b) na categoria de jatos regionais, por outro lado, o maior destaque foi para os jatos F 70 e F 100 da Fokker; c) na categoria narrow-body, mais se destacaram as empresas Boeing, com os modelos B 757-200F e B 757-200, e Tupolev, com o modelo TU 154M; d) e, por fim, na categoria wide-body, o destaque absoluto foi o modelo MD 11F, da McDonnell Douglas. Vale mencionar a ausência da fabricante Airbus da lista das aeronaves mais eficientes, sendo que alguns de seus aviões, como o A 380-800 e o A 300-600ST (Beluga), figuraram na lista dos piores, quanto a custo/benefício.

De maneira geral, as DMUs mais eficientes foram aviões de produção interrompida e com tecnologia não defasada, pois contam com um bom preço de mercado, um razoável custo de operação e benefícios semelhantes aos de um avião ainda em produção. Os aviões tecnologicamente avançados e que ainda estão sendo produzidos, por sua vez, tendem a não atingir a fronteira de eficiência porque possuem um alto preço de mercado, sendo que, embora a incorporação de novas tecnologias melhore os índices de output, o alto preço de compra acaba comprometendo o custo/benefício deste tipo de avião. Por fim, os aviões tecnologicamente defasados e de produção interrompida foram os que obtiveram os piores indices de desempenho, pois embora tenham um baixo preço de compra, são detentores de altos custos operacionais e baixo desempenho, pois consomem mais combustíveis, carregam menos carga, apresentam limitações de velocidade e são menos seguros. 
Tabela 1. Eficiência composta de aviões turboélices.

\begin{tabular}{|c|c|c|c|c|c|c|c|}
\hline \multirow{2}{*}{ Aviões } & \multicolumn{3}{|c|}{$\mathrm{km}$} & \multirow{2}{*}{ Aviões } & \multicolumn{3}{|c|}{$\mathrm{km}$} \\
\hline & 500 & 1000 & 1500 & & 500 & 1000 & 1500 \\
\hline AN32 & 0,86 & 0,86 & 0,86 & FD 228-212 & 0,78 & 0,77 & 0,85 \\
\hline ATR 42-320 & 0,79 & 0,82 & 0,90 & FD 328 & 0,85 & 0,83 & 0,86 \\
\hline ATR 42-500 & 0,81 & 0,81 & 0,86 & Jetstream 32EP & 0,79 & 0,79 & 0,84 \\
\hline ATR 72-500 & 0,81 & 0,76 & 0,79 & Jetstream 41 & 0,65 & 0,70 & 0,76 \\
\hline Beech 1900D & 1 & 1 & 1 & L 100-30 Hercules & 0,81 & 0,81 & 0,86 \\
\hline Beech King Air 1900 & 0,81 & 0,81 & 0,86 & LET 420 & 0,81 & 0,81 & - \\
\hline Dash 8-100A & 0,98 & 0,97 & - & Saab 2000 & 0,81 & 0,81 & 0,91 \\
\hline Dash 8-200A & 0,80 & 0,82 & 0,88 & Saab 340B+ & 0,75 & 0,73 & 0,77 \\
\hline Dash 8-300A & 0,89 & 0,91 & - & $Y-7-100$ & 0,81 & 0,81 & - \\
\hline Dash 8-400A & 0,82 & 0,82 & 0,86 & Y-7-200A & 0,67 & 0,70 & 0,75 \\
\hline EMB 120ER Brasília & 0,74 & 0,78 & 0,86 & & & & \\
\hline
\end{tabular}

Tabela 2. Eficiência composta de jatos regionais.

\begin{tabular}{|c|c|c|c|c|c|}
\hline \multirow{2}{*}{ Aviões } & \multicolumn{2}{|c|}{$\mathrm{km}$} & \multirow{2}{*}{ Aviões } & \multicolumn{2}{|c|}{$\mathrm{km}$} \\
\hline & 1000 & 2500 & & 1000 & 2500 \\
\hline Avro RJ 100 & 0,80 & - & DC 9-40 & 0,80 & - \\
\hline Avro RJ 70 & 0,73 & - & DC 9-50 & 0,80 & 0,89 \\
\hline Avro RJ 70ER & - & 0,89 & E 170 STD & 0,74 & 0,86 \\
\hline Avro RJ 85 & 0,80 & - & E 175 STD & 0,75 & 0,85 \\
\hline B 717-200 HGW & 0,80 & 0,82 & ERJ 135 ER & 0,75 & - \\
\hline BAC 1-11 475 & - & 0,89 & ERJ 140 ER & 0,83 & - \\
\hline BAC 1-11 500 & 0,80 & 0,89 & ERJ 145 ER & 0,80 & - \\
\hline BAe $146-100$ & 0,93 & - & ERJ $145 \mathrm{XR}$ & - & 0,89 \\
\hline BAe 146-200 & 0,94 & - & F 100 & - & 1 \\
\hline BAe $146-300$ & 0,82 & - & F 28-4000 & 0,80 & 0,89 \\
\hline CRJ 100 & 0,83 & - & F 70 & 1 & 0,99 \\
\hline CRJ 200 & 0,80 & 0,93 & FD $328 \mathrm{JET}$ & 0,80 & - \\
\hline CRJ 200 ER & 0,80 & - & MD 82 & - & 0,91 \\
\hline CRJ 200 LR & 0,80 & 0,89 & MD 88 & - & 0,84 \\
\hline CRJ 705 & 0,80 & 0,90 & TU 204 & - & 0,89 \\
\hline DC 9-30 & - & 0,88 & Yak 42 & 0,56 & - \\
\hline
\end{tabular}

Tabela 3. Eficiência composta de aviões Narrow-body.

\begin{tabular}{|c|c|c|c|c|c|c|c|}
\hline \multirow{2}{*}{ Aviões } & \multicolumn{3}{|c|}{$\mathrm{km}$} & \multirow{2}{*}{ Aviões } & \multicolumn{3}{|c|}{$\mathrm{km}$} \\
\hline & 2500 & 4000 & 5500 & & 2500 & 4000 & 5500 \\
\hline A 300 B4-200 & 0,78 & 0,80 & 0,56 & B 737-900 & 0,70 & 0,78 & - \\
\hline A $300-600$ & 0,64 & 0,67 & 0,64 & B 737-900ER & 0,76 & 0,81 & 0,81 \\
\hline A 300-600ST (Beluga) & 0,57 & 0,40 & 0,33 & B 757-200 & 0,74 & 0,89 & 1 \\
\hline A $318-100 \mathrm{HGW}$ & 0,87 & 0,90 & 0,71 & B 757-200F & 0,98 & 1 & 0,92 \\
\hline A $319-100$ & 0,65 & 0,73 & 0,71 & B 757-200HGW & - & 0,76 & 0,81 \\
\hline A $319-100 \mathrm{HGW}$ & 0,64 & 0,68 & 0,71 & В 757-300 & 0,84 & 0,92 & 0,93 \\
\hline A $320-200$ & 0,77 & 0,78 & 0,79 & В 777-200 & 0,72 & 0,73 & 0,71 \\
\hline A $320-200 H G W$ & 0,74 & 0,75 & 0,75 & CRJ 900 & 0,72 & 0,73 & - \\
\hline A $321-200$ & 0,73 & 0,74 & - & E 190 STD & 0,79 & 0,81 & - \\
\hline B 727-200 & 0,72 & 0,73 & - & E 195 STD & 0,68 & 0,69 & - \\
\hline В 737-200 & 0,52 & 0,52 & - & MD 81 & 0,81 & 0,77 & - \\
\hline В $737-300$ & 0,78 & 0,78 & - & MD 83 & 0,81 & 0,91 & - \\
\hline B 737-400 & 0,87 & 0,91 & - & MD 87 & 0,97 & 0,92 & - \\
\hline B 737-500 & 0,75 & 0,73 & - & MD 90-30 & 0,81 & 0,81 & - \\
\hline В 737-600 & 0,75 & 0,85 & 0,71 & TU 154M & 1 & 0,96 & - \\
\hline В $737-700$ & 0,73 & 0,82 & 0,78 & Yak 42M & 0,72 & 0,73 & - \\
\hline В $737-800$ & 0,82 & 0,87 & - & & & & \\
\hline
\end{tabular}


Tabela 4. Eficiência composta de aviões Wide-body.

\begin{tabular}{|c|c|c|c|c|c|c|}
\hline \multirow{2}{*}{ Aviões } & \multicolumn{6}{|c|}{$\mathrm{km}$} \\
\hline & 4000 & 5500 & 7000 & 8500 & 10000 & 11550 \\
\hline A $300-600 R$ & 0,68 & 0,64 & 0,65 & 0,71 & - & - \\
\hline A $310-200$ & 0,71 & 0,65 & 0,60 & - & - & - \\
\hline A $310-300$ & 0,68 & 0,72 & 0,67 & - & - & - \\
\hline A $330-200$ & - & 0,65 & 0,68 & 0,74 & 0,84 & 0,79 \\
\hline A $330-300$ & 0,77 & 0,63 & 0,59 & 0,59 & 0,69 & - \\
\hline A $340-200$ & - & 0,68 & 0,71 & 0,77 & 0,83 & 0,79 \\
\hline A $340-300$ & - & 0,69 & 0,72 & 0,76 & 0,81 & 0,79 \\
\hline A $340-500$ & - & - & 0,59 & 0,61 & 0,68 & 0,72 \\
\hline A $340-600$ & - & 0,70 & 0,69 & 0,70 & 0,73 & 0,73 \\
\hline A $380-800$ & - & - & 0,45 & 0,45 & 0,51 & 0,59 \\
\hline A $380-800 F$ & - & 0,72 & 0,68 & 0,68 & 0,73 & 0,79 \\
\hline AN $124-100$ & 0,77 & 0,66 & 0,55 & 0,52 & - & - \\
\hline B 747-100 & 0,68 & 0,63 & 0,61 & 0,59 & 0,62 & - \\
\hline B 747-200 & 0,90 & 0,81 & 0,75 & 0,73 & 0,73 & 0,71 \\
\hline B 747-200F & 0,90 & 0,91 & 0,88 & 0,85 & 0,89 & 0,83 \\
\hline В 747-300 & - & 0,84 & 0,83 & 0,81 & 0,83 & 0,79 \\
\hline В $747-400$ & - & 0,74 & 0,81 & 0,81 & 0,88 & 0,91 \\
\hline B 747-400F & - & 0,82 & 0,84 & 0,84 & 0,90 & 0,93 \\
\hline B 747-8 1ntercontinental & - & - & 0,68 & 0,68 & 0,73 & 0,79 \\
\hline B 747SP & - & - & 0,70 & 0,72 & 0,84 & 0,79 \\
\hline B 767-200 & 0,77 & 0,72 & 0,68 & - & - & - \\
\hline B 767-200ER & 0,77 & 0,72 & 0,73 & 0,81 & 0,88 & - \\
\hline В 767-300 & 0,68 & 0,60 & 0,58 & 0,68 & - & - \\
\hline B 767-300ER & 0,88 & 0,81 & 0,75 & 0,75 & - & - \\
\hline B 767-400ER & 0,77 & 0,72 & 0,68 & 0,66 & 0,73 & - \\
\hline B 777-200LR & - & - & 0,65 & 0,68 & 0,75 & 0,83 \\
\hline В 777-300 & 0,77 & 0,57 & 0,55 & 0,51 & 0,53 & 0,58 \\
\hline B 777-300ER & - & 0,72 & 0,72 & 0,74 & 0,69 & 0,67 \\
\hline B 787-8 Dreamliner & - & 0,72 & 0,72 & 0,79 & 0,89 & 0,98 \\
\hline DC $10-10$ & 0,86 & 0,72 & 0,63 & 0,64 & - & - \\
\hline DC $10-30$ & - & 0,83 & 0,83 & 0,89 & 0,94 & - \\
\hline DC $10-40$ & 0,71 & 0,70 & 0,68 & 0,68 & 0,73 & - \\
\hline $1162 \mathrm{MK}$ & 0,77 & 0,72 & 0,68 & 0,68 & - & - \\
\hline $1196-300$ & 0,77 & 0,72 & 0,68 & 0,68 & - & - \\
\hline L 1011-1 Tristar & 0,77 & 0,72 & 0,68 & - & - & - \\
\hline L 1011-100 Tristar & 0,77 & 0,72 & 0,71 & - & - & - \\
\hline L 1011-200 Tristar & 0,77 & 0,72 & 0,75 & 0,70 & - & - \\
\hline L 1011-250 Tristar & 0,85 & 0,80 & 0,81 & 0,81 & 0,73 & - \\
\hline L 1011-500 Tristar & 0,91 & 0,86 & 0,87 & 0,84 & 0,73 & - \\
\hline MD 11 & - & 0,91 & 0,91 & 0,94 & 0,98 & 0,95 \\
\hline MD $11 \mathrm{~F}$ & 1 & 1 & 1 & 1 & 1 & 1 \\
\hline
\end{tabular}

Quadro 4. Aviões a jato mais e menos eficientes.

\begin{tabular}{|c|c|c|c|c|}
\hline Propulsão & Categoria & Alcance $(\mathrm{km})$ & Aeronave eficiente & Aeronave menos eficiente \\
\hline \multirow{3}{*}{ Turboélice } & \multirow{3}{*}{ Regional } & 500 & Beech 1900D & Jetstream 41 \\
\hline & & 1000 & Beech 1900D & Jetstream 41 \\
\hline & & 1500 & Beech 1900D & Y-7-200A \\
\hline \multirow{11}{*}{ Jato } & \multirow{2}{*}{ Regional } & 1000 & F 70 & Yak 42 \\
\hline & & 2500 & F 100 & B 717-200 HGW \\
\hline & \multirow{3}{*}{ Narrow-body } & 2500 & TU 154M & B 737-200 \\
\hline & & 4000 & B 757-200F & A 300-600ST (Beluga) \\
\hline & & 5500 & B 757-200 & A 300-600ST (Beluga) \\
\hline & \multirow{6}{*}{ Wide-body } & 4000 & MD $11 \mathrm{~F}$ & B 767-300 \\
\hline & & 5500 & MD 11F & B 777-300 \\
\hline & & 7000 & MD 11F & A 380-800 \\
\hline & & 8500 & MD 11F & A $380-800$ \\
\hline & & 10000 & MD 11F & A $380-800$ \\
\hline & & 11500 & MD 11F & B 777-300 \\
\hline
\end{tabular}




\section{Conclusão}

Pelo que se pôde verificar nesta pesquisa, a utilização da DEA foi bastante satisfatória para analisar a relação custo/beneficio de aeronaves, sendo que de maneira relativamente simples e rápida, foi estabelecido, para diferentes categorias, 0 ranking das aeronaves com melhor custo/beneficio.

Foram obtidas, com a DEA, conclusões bastante interessantes sobre a relação custo/ benefício de aeronaves como, por exemplo, o fato dos aviões mais eficientes serem os de produção interrompida e com tecnologia não defasada. Essa constatação parece refletir a estratégia da indústria aeronáutica de lançar produtos cada vez mais cedo, com altos preços de mercado, sem que ainda exista um incremento de tecnologia com valor percebido suficiente para que esses preços sejam compensadores, em termos de custo/benefício, para um comprador. Vale ressaltar que, para que esses novos produtos cheguem ao mercado, os produtos antigos precisam ser tirados de circulação, apesar de ainda interessarem aos clientes.

A metodologia aplicada neste artigo possuiu algumas limitações como, por exemplo, o fato de todos os inputs e outputs terem tido a mesma importância na análise, sendo que não foi considerado o fato de que os compradores podem considerar alguns inputs ou outputs mais relevantes do que os outros na hora de tomarem sua decisão.

Como sugestão para trabalhos futuros poderse-ia utilizar a DEA com restrições de peso, com o objetivo de levar em conta, na análise, as diferenças de importância entre inputs e outputs para os compradores. Para que essa diferença de importância pudesse ser determinada, seria necessária uma pesquisa qualitativa, que poderia ser realizada com o auxílio da Técnica do Incidente Crítico (TIC) e da Análise Conjunta, que são ferramentas desenvolvidas pela área de marketing. Outra sugestão para trabalhos futuros seria comparar os resultados deste artigo com outros métodos de análise de custo/beneficio de aeronaves, tais como as técnicas multicritério de apoio à decisão e a lógica fuzzy.

\section{Referências}

ABBOTT, M.; WU, S. Total factor productivity and efficiency of Australian airports. The Australian Economic Review, v. 35, n. 3, p. 244-260, 2002. http://dx.doi.org/10.1111/14678462.00241

AIRBUS. Aircraft families. Disponível em: <www.airbus.com>. Acesso em: 15 set. 2009
ALMEIDA, M. R.; MARIANO, E. B.; REBELATTO, D. A. N. Análise de eficiência de aeroportos internacionais brasileiros. Revista Produção Online, v. 7, p. 9-26, 2008.

ANGULO-MEZA, L. et al. lsyds - integrated system for decision support (SIAD - Sistema Integrado de Apoio a Decisão): a software package for data envelopment analysis model. Pesquisa Operacional, v. 25, n. 3, p. 493-503, 2005.

ANGULO-MEZA, L.; LINS, M. P. E. Review of methods for increasing discrimination in data envelopment analysis. Annals of Operations Research, v. 116, n. 1-4, p. 225-242, 2002. http://dx.doi.org/10.1023/A:1021340616758

AVION SAFETY NETWORK. Database. Disponivel em: <www. aviation-safety.net>. Acesso em: 15 set. 2009.

BANKER, R. D.; CHARNES, A.; COOPER, W. W. Some models for estimating technical and scale inefficiencies in data envelopment analysis. Management Science, v. 30, p. 1078-1092, 1984. http://dx.doi.org/10.1287/mnsc.30.9.1078

BARROS, C. P.; PEYPOCH, N. An evaluation of European airlines operational performance. International Journal of Production Economics, v. 122, n. 2, p. 525-533, 2009. http://dx.doi.org/10.1016/j.jpe.2009.04.016

BAZARGAN, M.; VASIGH, B. Size versus efficiency: a case study of US commercial airports, Journal of Air Transport Management, v. 9, p. 187-193, 2003. http://dx.doi. org/10.1016/S0969-6997(02)00084-4

BOEING. Commercial airplanes. Disponível em: <www.boeing. com>. Acesso em: 15 set. 2009.

BOMBARDIER. Products. Disponivel em: <www.bonbardier. com>. Acesso em: 15 set. 2009.

BONACORSI, A.; GIURI, P.; PIEROTTI, F. Technological frontier rs and competition in multi-technology sectors. Economics of innovation and new technology, v. 14, p. 23-42, 2005. http://dx.doi.org/10.1080/1043859042000228660

CHARNES, A.; COOPER, W. W.; RHODES, E. Measuring the efficiency of decision making units. European Journal of Operational Research, v. 2, p. 429-444, 1978. http://dx.doi. org/10.1016/0377-2217(78)90138-8

COOPER, W.; SIEFORD, L.; TONE, K. Date envelopment analysis. A comprehensive text with models, applications, reference and DEA-Solver software. Boston: Kluwer Academic Publishers, 2000.

CORREIA, T. C. V. D.; SOARES DE MELLO, J. C. C. B. Avaliação da eficiência das companhias aéreas brasileiras com modelo DEA nebuloso. In: Associação Nacional de Pesquisa e Ensino em Transporte (Org.) Transporte em transformação XIII: trabalhos vencedores do Prêmio CNT Produção Acadêmica 2009. Brasília: Gráfica Positiva, 2009. p. 199-215.

DISTEXHE, V.; PERELMAN, S. Technical efficiency and productivity growth in an era of deregulation: the case of airlines. Swiss Journal of Economics and Statistics, v. 130, n. 4, p. 669-689, 1994.

EMBRAER. Aeronaves. Disponivel em: <www.embraer.com>. Acesso em: 15 set. 2009.

FlELDING, J. P. Introduction to aircraft design. 4. ed. New York: Cambridge University Press, 2003.

G00D, D. et al. Airline efficiency differences between Europe and the US: implications for the pace of EC integration and domestic regulation. European Journal of Operational Research, v. 80, p. 508-518, 1995. http://dx.doi. org/10.1016/0377-2217(94)00134-X

GUEDES, E. C. C.; SANTOS, R. P.; CORREIA, A. R. Airport efficiency: a comparative approach with data envelopment analysis and inverted frontier. In: ANNUAL WORLD CONFERENCE - AIR TRANSPORT RESEARCH SOCIETY ATRS, 10., 2006, Nagoya. Anais... 
HIGGINS, K. M.; LAWPHONGPANICH, S.; MAHONEY, J. F. Evaluating airline service quality by data envelopment analysis. Transportation Research Record, v. 2052, p. 1-8, 2008. http://dx.doi.org/10.3141/2052-01

KUOSMANEN, T.; BIJSTERBOSCH, N.; DELLINK, R. Environmental cost-benefit analysis of alternative timing strategies in greenhouse gas abatement: a data envelopment analysis approach. Ecological Economics, v. 68, n. 6, p. 1633-1642, 2009.

LAM, S. W.; LOW, J. M. W.; TANG, L. C. Operational efficiencies across Asia Pacific airports. Transportation resarch part E-Logistics and transportation review, v. 45, n. 4, p. 654-665, 2009.

LETA, F. R. et al. Métodos de melhora de ordenação em DEA aplicados à avaliação estática de tornos mecânicos. Investigação Operacional, v. 25, n. 2, p. 229-242, 2005.

LIN, L. C.; HONG, C. H. Operational performance evaluation of international major airports: an application of data envelopment analysis. Journal of Air Transport Management, v. 12, n. 6, p. 342-351, 2006. http://dx.doi. org/10.1016/j.jairtraman.2006.08.002

MARTIN, J. C.; ROMAN, C. A benchmarking analysis of Spanish commercial airports. A comparison between SMOP and DEA ranking methods. Network \& Spatial Economics, v. 6 , n. 2, p. 111-134, 2006. http://dx.doi.org/10.1007/s11067006-7696-1

MAYSTRE, L. Y.; PICTET, J. E.; SIMOS, J. Méthodes multicritères ELECTRE: description, conseils pratiques et cas d'application à la gestion environnementale. Lausanne: Presses Polytechniques et Universitaires Romandes, 1994.

PACHECO, R. R.; FERNANDES,, E. Managerial efficiency of Brazilian airports. Transportation research part A-policy and practice, v. 37, n. 8, p. 667-680, 2003. http://dx.doi. org/10.1016/S0965-8564(03)00013-2

PADILHA, C. E. Optimizing Jet transport Efficiency. New York: McGraw-Hill Companies, 1996.

PARKER, D. The performance of BAA before and after privatization-A DEA study. Journal of Transport Economics and Policy, v. 33, n. 2, p. 133-146, 1999.

RANGEL, L. A. D.; GOMES, L. F. A. M. 0 apoio multicritério à decisão na avaliação de candidatos. Produção, v. 20, n. 1, p. 92-111, 2010. http://dx.doi.org/10.1590/S010365132010005000016

RAY, S. C. The directional distance function and measurement of super-efficiency: an application to airlines data. Journal of Operational Research Society, v. 59, n. 6, p. 788-797. 2008. http://dx.doi.org/10.1057/palgrave.jors.2602392

RISINGUP AVIATION RESOURCES. Databases. Disponível em: $<$ www.risingup.com>. Acesso em: 15 set. 2009.

ROGHANIAN, E.; FOROUGHI, A. An empirical study of Iranian regional airports using robust data envelopment analysis. International Journal of Industrial Engineering Computations, v. 1, p. 65-72, 2010. http://dx.doi. org/10.5267/j.jjiec.2010.01.006

ROSKAM, J. Airplane Flight Dynamics - Part VIIl: airplane cost estimation: design, development, manufacturing and operating. Ottawa: Roskam Aviation and Engineering, 1979.

SENRA, L. F. A. C.; et al. Estudo sobre métodos de seleção de variáveis em DEA. Pesquisa Operacional, v. 27, n. 2, p. 191-207, 2007.

SILVEIRA, J. 0. et al. Avaliação da eficiência das companhias aéreas brasileiras com uma variação do modelo de $\mathrm{Li}$ e Reeves. Engevista, v. 10, n. 2, p. 145-155, 2008.

SOARES DE MELLO, J. C. C. B. et al. Análise de envoltória de dados no estudo da eficiência e dos benchmarks para companhias aéreas brasileiras. Pesquisa Operacional, v. 23, n. 2, p. 325-345, 2003.

SOARES DE MELLO, J. C. C. B. et al. DEA advanced models for geometric evaluation of used lathes. WSEAS Transactions on Systems, v. 7, n. 5, p. 500-520, 2008.

WANG, T. C.; CHANG, T. H. Application of TOPISIS in evaluating initial training aircraft under a fuzzy environment. Expert Systems With Applications, v. 33, n. 4, p. 870-880, 2007. http://dx.doi.org/10.1016/j.eswa.2006.07.003

WHEL. Commercial aircraft. Disponível em: <www.whel.de>. Acesso em: 15 set. 2009.

WOMER, N. K. et al. Benefit-cost analysis using data envelopment analysis. Annals of Operations Research, v. 145 , n. 1, p. 229-250, 2006. http://dx.doi.org/10.1007/ s10479-006-0036-5

YOSHIDA, Y.; FUJIMOTO, H. Japanese-airport benchmarking with the DEA and endogenous-weight TFP methods: testing the criticism of overinvestment in Japanese regional airports. Transportation Resarch Part E-Logistics and Transportation Review, v. 40, n. 6, p. 533-546, 2004. http://dx.doi.org/10.1016/j.tre.2004.08.003

YU M. M. Capacity efficiency measurement using a three-stage DEA approach: evidence from domestic airports in Taiwan. TransportationPlanningandTechnology,v.33,n.2,p.221-235, 2010. http://dx.doi.org/10.1080/03081061003643804

\section{Cost-benefit of aircrafts: an approach through Data Envelopment Analysis}

\section{Abstract}

The objective of this paper was to apply the operational research technique of Data Envelopment Analysis (DEA), through the composite index of classic and inverted borders, to evaluate the cost-benefit of civil transport aircrafts. To this end, a) market price, and b) operating costs were used as inputs; and, a) payload, b) cruise speed, and c) maximum reason climb with one engine were used as outputs. To ensure homogeneity of DMUs, the planes were divided according to the propulsion system and in the following categories: regional, narrow-body and wide-body; they were also grouped into different ranges, so that the best cost-benefit for each aircraft could be identified. Results allowed for the following conclusion: the best cost-benefits were found in planes where the production had already been discontinued, but they were not very technologically outdated yet. Keywords Civil transport aircraft. Cost-benefit analysis. Data Envelopment Analysis - DEA. Inverted frontier. 